\title{
Telemedicine for Chronic Digestive Diseases: A Systematic Qualitative Review
}

\author{
Sandra M. Quezada ${ }^{1}$ and Raymond K. Cross ${ }^{1,2}$ \\ ${ }^{1}$ University of Maryland School of Medicine \\ Department of Medicine, Division of Gastroenterology and Hepatology \\ ${ }^{2}$ Veterans Affairs Maryland Health Care System \\ USA
}

\section{Introduction}

According to 2004 statistics from the National Digestive Diseases Information Clearinghouse, digestive diseases affect 60-70 million people in the United States, resulting in 105 million ambulatory visits and 13.5 million hospitalizations. The cost burden associated with these diseases is high. It is estimated that direct and indirect medical costs related to digestive diseases are $\$ 98$ billion and $\$ 44$ billion, respectively. Chronic digestive diseases make up a significant proportion of these disorders, including but not limited to celiac disease, chronic constipation, chronic pancreatitis, cirrhosis, Crohn's disease, gastroesophageal reflux disease, irritable bowel syndrome, ulcerative colitis, and viral hepatitis.

Telemedicine has been used successfully in chronic conditions such as asthma, diabetes, and congestive heart failure. In patients with asthma, telemedicine improves symptoms, decreases use of quick relief inhalers, improves adherence with self-action plans, improves quality of life and patient knowledge, and decreases urgent care visits (Joshi et al, 2005). In diabetes, telemedicine reduces glycosylated hemoglobin levels (Quinn et al, 2009). Telemedicine improves quality of life, and decreases hospitalizations and costs in congestive heart failure (Roth et al, 2004).

Telemedicine has proven to be a feasible and well accepted method of treatment delivery in the field of gastroenterology as well. A systematic review of scientific publications was performed in order to identify all studies conducted examining the application of telemedicine in digestive diseases. Database searches in MEDLINE, the Cochrane Controlled Trials Register, and Web of Science Conference Proceedings Citation Index were done with the following search terms: telemedicine, gastroenterology, inflammatory bowel disease, ulcerative colitis, Crohn's disease, gastroesophageal reflux disease, hepatitis $C$, hepatitis B, chronic liver disease, cirrhosis, constipation, irritable bowel syndrome, microscopic colitis, celiac disease and chronic pancreatitis. This search yielded telemedicine studies conducted in one of three disease states: inflammatory bowel disease with a specific focus in ulcerative colitis, irritable bowel syndrome, and chronic viral hepatitis C. In this chapter, we will review the use of telemedicine for these chronic digestive diseases and the effect of telemedicine on access to care, disease activity, education, and quality of life. 


\section{Inflammatory bowel disease}

Many of the telemedicine studies in digestive diseases focuses on the chronic disease model of inflammatory bowel disease (IBD). IBD is comprised of ulcerative colitis (UC) and Crohn's disease (CD), two inflammatory conditions of the intestines that affect approximately 1.4 million Americans, with that number evenly split between the two conditions (Crohn's and Colitis Foundation of America [CCFA], 2010). Symptoms of UC and $\mathrm{CD}$ can be quite debilitating, most commonly including diarrhea (which can be bloody) and abdominal pain. Typically, symptoms are chronic and characterized by periods of remission interrupted by exacerbations.

Effective medical therapy for induction and maintenance of remission exists for both UC and $\mathrm{CD}$; however, the relatively young age at diagnosis, potential for medication side effects, and the long-term need for medication to maintain remission can result in decreased adherence, which places patients at risk for exacerbations of disease (Kane et al., 2003). Also, patient miscomprehension can lead to decreased medication adherence (Sewitch, et al., 2003) and delays in seeking medical care with associated adverse outcomes.

Several studies have evaluated the use of telemedicine in the IBD population. One pilot study assessed the acceptance of a home telemanagement system (HAT) in ten patients with IBD (Cross et al., 2006). This study utilized a HAT system developed for patients with asthma and other chronic respiratory diseases (Finkelstein et al, 2001 as cited in Cross et al, 2006). IBD HAT is comprised of three components: a patient home unit, a decision support server, and a web-based clinician portal as depicted in Figure 1.

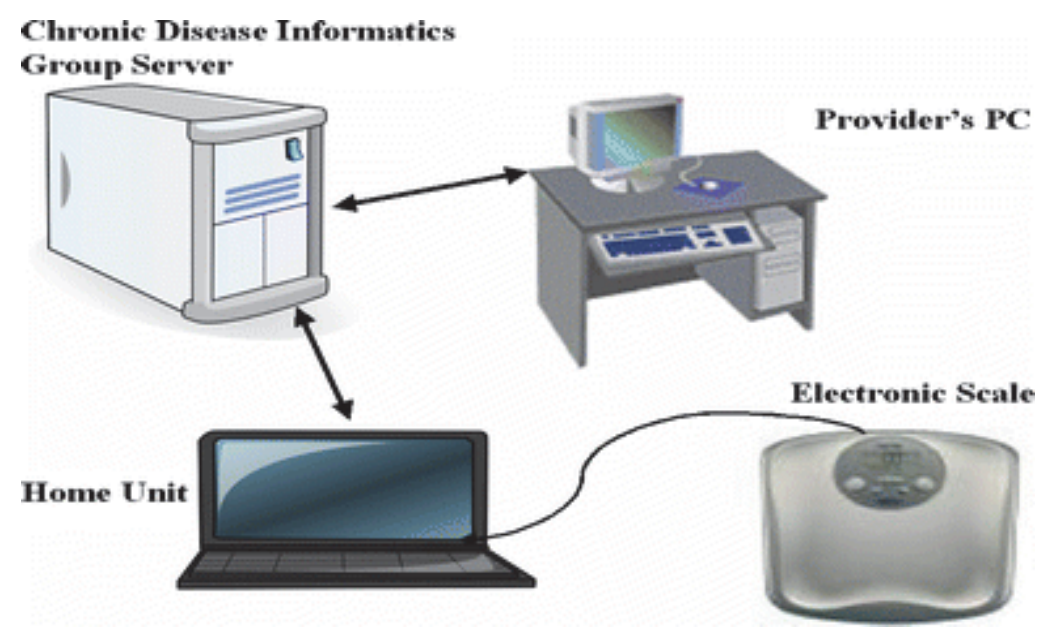

Fig. 1. Model of the home telemanagement system for inflammatory bowel disease

The patient home unit includes an electronic weight scale connected to a laptop computer via a serial port for self-testing. The laptop computer contains a symptom diary, side effect inventory, adherence check, and assessment of body weight. Patients answer questions directly using the laptop; weight is assessed after audio prompts from the laptop. Individualized patient data is entered into the secure web portal; information collected includes contact information, medication prescriptions, HAT testing schedules, and disease history. Clinicians use the web portal to customize medication and side effect profiles. 
Furthermore, a clinical alert system is customized for each patient based on responses to the symptom diary, medication side effect questions, self-reported adherence, and body weight. Once self-testing is completed, patients received an IBD-related educational prompt in the format of a "tip of the day"; the following week, patients are asked a question about the tip. Patients cannot advance in the educational curriculum unless they answer the question correctly. The results of self-testing are submitted telephonically and are available for review immediately on the secure web server.

Five participants in this study were diagnosed with $C D$ and five were diagnosed with UC. The mean disease duration was 15 years. Participant average age was 47 years. Ninety percent of participants were Caucasian, with mean years of education of 15 years. All reported excellent English proficiency. Thirty percent reported limited knowledge of IBD. Two participants had never used a home computer and one had never used the Internet.

All participants found the self-test procedures to be uncomplicated and reported no difficulty using the computer or answering the symptom diary and side effect questions. Ninety percent reported no difficulty with using the weight scale. All patients felt the selftesting procedures took very little time and felt it would result in very little to no interference with their daily activities. Thirty percent would feel significantly or moderately safer while using this system. Seventy percent of participants reported that testing up to four times per week was feasible; all participants felt that weekly testing was realistic.

Based on their results, the authors concluded that patients with IBD accepted IBD HAT technology and that use of IBD HAT was feasible in the IBD population.

A follow-up analysis was performed to assess acceptance and feasibility of the home telemanagement system among 34 participants with IBD (Cross \& Finkelstein, 2007). After an initial 40 minute training period, participants were asked to complete weekly self-testing for six months. Disease activity, quality of life and IBD knowledge were assessed at baseline and at six months. Seven participants were excluded, due to either lack of adherence with home installation of the system or lack of a telephone line. Overall, weekly adherence to self-testing over the 6 month study was 91\%. Once again, participants reported that IBD HAT was easy to use and did not interfere with their daily activities. In addition, use of IBD HAT positively impacted participants' satisfaction with their IBD care, as $86 \%$ reported receiving excellent care after use of IBD HAT as compared with $65 \%$ of participants at baseline. Similarly, $71 \%$ reported having their needs met at the end of the six months as compared with $57 \%$ at baseline. Ninety percent reported that they were quite satisfied with their IBD care at 6 months compared with $70 \%$ initially. Patient knowledge also improved significantly after using the IBD HAT educational curriculum.

While this study was not powered to detect significant differences in disease activity and disease-specific quality of life, the authors reported strong trends toward improvement in these factors, as measured by the Harvey Bradshaw Index (HBI) for CD activity (a modified HBI was utilized for UC) (Harvey \& Bradshaw, 1980, as cited in Cross \& Finkelstein, 2007) and the Short Inflammatory Bowel Disease Questionnaire (SIBDQ) for disease-specific quality of life (Irvine et al, 1996, as cited in Cross \& Finkelstein, 2007).

Qualitative interviews were performed for 23 of the participants (Castro et al., 2006). Feedback from participants was categorized into one of three topics: 1) content of the system components, 2) interface between the system and the user, and 3) process of using the system. Participants reported that the greatest benefit of IBD HAT was the increased sense of empowerment and control over their IBD care as compared with regular monitoring. This resulted in improved patient satisfaction and outcomes. Overall, the authors concluded that 
use of IBD HAT over 6 months was feasible and that patient acceptance of the technology was high.

An abstract from the Digestive Diseases Week (annual conference of the American Gastroenterological Association) in May 2010 presented data on a prospective, randomized, controlled, pilot study, performed to evaluate the effectiveness and applicability of a novel telemedicine system in an IBD clinic (Krier et al., 2010). Newly established patients were randomized into one of two groups: 1) a remote clinical encounter via telemedicine with an IBD provider (located 33 miles away) or 2) a conventional in-person encounter with an IBD provider. The telemedicine system consisted of two standard Apple computers running a secure protocol between the subspecialist and the participant. Fifteen participants were enrolled (8 with UC, 7 with CD). Eighty percent of participants were men, with a median age of 60 years and median disease duration of 7 years. Participant experience was rated using the Ware Specific Visit Standard Questionnaire $(1=$ excellent, $5=$ poor $)$. Participants in both groups rated their clinic experience highly $(1.2 \pm 0.4$ in the telemedicine group versus $1.1 \pm 0.4$ in the conventional group). Both remote and in-person clinic visits were of similar duration (58 minutes in the telemedicine group versus 53 minutes in the conventional group). High ratings on the technical and informational quality of the telemedicine sessions were reported from the providers involved. The authors concluded that telemedicine can be used successfully to increase access to quaternary care in large health care systems, resulting in patient satisfaction similar to face-to-face clinical encounters.

In summary, the above studies demonstrate that patients with IBD reported a high level of acceptance of telemedicine as a means of receiving care. Telemedicine positively impacted participants' perception of their care and gave them a sense of empowerment, as it encouraged them to actively participate in their disease management. The relatively low numbers of participants in the initial studies limited the ability of the investigator's to evaluate for differences in disease activity, quality of life, or utilization of health care resources. However, the results supported the need for further investigation in this field with larger sample sizes of patients with IBD.

\subsection{Ulcerative colitis}

As noted earlier, IBD is comprised of two distinct disease entities UC and CD. While the two disease processes are similar, important symptomatic and prognostic distinctions exist between the two. For example, while both CD and UC cause diarrhea, the diarrhea in UC tends to be bloody, while in CD it is not. In addition, CD can be complicated by intestinal stricture, fistula formation, and perianal disease involvement. Thus, measuring symptoms and disease activity of both diseases collectively misrepresents the unique symptomatic characteristics of each. Telemedicine has been studied primarily in the UC population.

In 2009, Cross et al. tested the feasibility and acceptance of telemedicine in ten patients with UC (Cross et al, 2009), with certain modifications to the IBD HAT system described previously (Cross et al, 2007). First, questions in the symptom diary were altered to be specific for UC. The UC symptom diary consists of 14 questions which assessed overall well being, functional status, bowel symptoms, systemic symptoms, and extraintestinal manifestations of UC. Responses were scored from a minimum of one to a maximum of five, yielding an overall minimum score of 14 and maximum score of 51 . An alert was generated for a score of 25 or higher. Subscores from 4 to 15 were also generated for a group of questions felt to be critically important that dealt with overall well-being, number of liquid stools per day, nocturnal awakening, and amount of visible blood in bowel movements. In this instance a subscore of 8 or more generates an alert to the provider. Total and sub-score 
thresholds can be individualized for each participant to increase or decrease sensitivity. Likewise, the alert system was altered to coincide with the new symptom diary.

Action plans at the end of each self-testing session were developed for participants. Based on scores generated from the UC symptom diary, participants receive self-action or action plans in one of three categories: 1) Green zone, for patients with no to mild symptoms, 2) Yellow zone, for patients with moderate symptoms, or 3) Red zone, for patients with severe symptoms. Each severity zone lists several actions that providers can choose for participants to initiate as part of their self-management plan. These action plans can also be modified by the provider on the web portal as needed. Lastly, an electronic messaging system was added to the HAT system for UC (UC HAT) so that participants can communicate with the medical center. The messaging center allows for both automated and free text messaging.

Attitudinal surveys were done to pre-test the UC HAT system. Pre-testing yielded similar results in the UC population compared to the overall IBD population. All participants felt that using the computer and self-testing system was not complicated, and nine of the ten participants reported no difficulty in using the weight scale or in answering the symptom diary and side effect questions. Seven participants reported that they would feel safer using UC HAT, and eight felt it was important that the IBD center physicians monitored their results. Participants also expressed a sense of improved communication, knowing the provider would be informed of their flares immediately. This improved communication, along with an increased sense of self-awareness, resulted in participants feeling empowered. Some participants reported that the self-testing process could replace clinic visits, although one participant stated that "certain things would be best discussed in person". There was some negative feedback about the UC HAT system as well. One participant felt the system was not tailored to him or her, and another felt that the system inaccurately overestimated the severity of his or her symptoms. Overall, the investigators felt that the UC HAT system was well received in this population and anticipated that its use would result in improved clinical outcomes as compared with routine care in patients with UC.

Subsequently, Cross et al. designed a randomized controlled trial to test the hypothesis that the UC HAT system would decrease disease activity, increase quality of life, and improve medical adherence compared with best available care (Cross et al. 2011). Forty-seven participants with UC were randomized to receive either home telemanagement with the UC HAT system (25 participants) or best available care (22 participants). Participants in the UC HAT group underwent self-testing weekly. Participants in the best available care group underwent routine and as needed clinic and telephone follow up, received educational fact sheets about IBD and received self-action plans without reinforcement. Disease activity was measured by the Seo Index (Seo et al, 1992, as cited in Cross et al. 2011) and disease-related quality of life was measured by the Inflammatory Bowel Disease Questionnaire (IBDQ) (Guyatt et al., 1989, as cited in Cross et al. 2011).

Sixty-four percent of the participants were women, and 66\% were Caucasian. At baseline, 27\% of participants in the best available care group used immune suppressants compared to $56 \%$ in the UC HAT group $(p=0.05)$. Further, IBDQ scores at baseline were lower in UC HAT participants compared to the best available care group. During the trial, 8 participants withdrew in the UC HAT arm compared to 1 in the best available care arm. There was no difference in disease activity scores or remission rates between the treatment groups at 4, 8 and 12 months. After adjustment for baseline quality of life, disease activity scores decreased 12 points from baseline in the UC HAT arm $(\mathrm{p}=0.08)$ compared to 1 point in the best available care arm $(p=0.84)$. IBDQ scores increased in the UC HAT arm and remained stable in the best 
available care arm, though these differences were not significant at any time point post baseline. However, after adjustment for baseline disease knowledge, UC HAT participants were noted to have a 16-point improvement in quality of life scores from baseline compared to the best available care group $(p=0.04)$. Adherence was low in both groups at baseline; adherence improved in both groups over 12 months. No significant differences in adherence were noted between groups. These results suggest that telemedicine may decrease disease activity and increase quality of life in patients with UC despite the finding that self-reported adherence did not improve in the UC HAT group. The high attrition rate in the UC HAT arm was concerning; it is possible that a different telemedicine system, such as a web-based design, would improve retention rates in future trials.

A randomized controlled trial in Denmark and Ireland was conducted to assess UC patients' use of web-based home telemanagement (Elkjaer et al., 2010). Patients with mild-tomoderate UC were randomly assigned to either a web-group with disease-specific education and self-treatment or a control group that received standard care for 12 months. Outcomes of interest included feasibility of the telemanagement approach and its influence on participants' medication adherence, UC knowledge, quality of life, safety, and health care costs. Exclusion criteria included use of infliximab and immune suppressant therapy, narcotic dependence, previous IBD surgery or likelihood of surgery during the study period, 2 or more flares per year requiring high-dose steroid therapy, pregnancy, and breastfeeding.

This study used the "Constant Care" website (http://www.constant-care.dk). Each participant was given a unique username and password. Prior to using the website, all participants in the web-group, and their relatives, were given educational training with a 1.5-hour slide presentation on IBD etiology, pathology, anatomy, medical and surgical treatments, disease course, adherence, nutrition, mortality risk, colorectal cancer chemoprevention, pregnancy, and breastfeeding. Participants and family members also underwent a 1.5-hour training session in using the Constant Care website. Guidelines for indications on when to call the provider included having greater than six stools per day, daily rectal bleeding, rectal bleeding occurring between relapses, fever $>37.5$ degrees Celsius, heart rate $>90$ beats per minute, severe abdominal pain, symptoms persisting more than eleven days despite escalation of therapy, unexplained weight loss, and/or for any doubts or questions regarding the study.

Disease-specific quality of life was measured on the website with the SIBDQ, and the Simple Clinical Colitis Activity Index (SCCAI) was used to assess disease activity (Walmsey et al., 1998, as cited in Elkjaer et al., 2010). Symptoms were categorized in a similar manner as in the UC HAT study; quiescent-to-mild symptoms appeared as a green traffic light, moderate symptoms appeared as a yellow light, and highly active symptoms appeared as a red light. During symptom flare, participants were instructed to log onto the system daily to complete the SCCAI until their symptoms entered the green zone. Entry frequency was then reduced to once weekly until 4 weeks after the initial relapse. The SIBDQ was to be completed at the beginning and end of each relapse. Once in remission, participants were to log into the system once monthly until the next relapse occurred. If symptoms were entered such as rectal bleeding, 3 or more bowel movements daily, or nighttime bowel movements, the system recommended initiation of $4 \mathrm{gm}$ daily or more of 5-aminosalicylic acid (5-ASA) treatment for a total of 28 days. Participants were given the option to extend this treatment period by an additional 28 days if remission into the green zone was not achieved. 
Participants could also choose additional topical 5-ASA treatment and prednisolone, based on previous maximal extent of disease and participants' prior treatment experience.

All participants were to have study visits at baseline, six months, and 12 months. During each of these visits participants were asked to complete a series of questionnaires, including the SCCAI, SIBDQ, Crohn's Colitis Knowledge Score (CCKNOW) (Eadan JA et al., 1999, as cited in Elkjaer et al., 2010), SF-36 (Ware et al., 2002, as cited in Elkjaer et al., 2010), Hospital Anxiety and Depression Scale (Zigmond \& Snaith, 1983, as cited in Elkjaer et al., 2010), and Compliance Questionnaire. The Compliance Questionnaire was developed at Herlev Hospital in Denmark, and it included 5 questions on the following topics: ease of access to prescription, ability of relapse recognition, following the medical doctor's advice, ability to self-initiate acute treatment, and adherence to 5-ASA treatment.

In total, 333 participants, aged 18-to-69 years, from both Danish and Irish sites were randomized. Of these, 135 participants completed the 12-month follow-up visit. The control group was predominantly female (70\% in control group vs. $51 \%$ in web-based group) and older (median age of 48 years in the control group compared with 41 years in the web group). Otherwise, there were no significant differences between the two randomized groups in terms of age at diagnosis, disease duration, disease extent, 5-ASA medication history, smoking status, marital status, education, or occupation.

In the Danish arm of the study, there were no differences in 5-ASA adherence, as measured by medication refills from the e-prescription pharmacy database, between the web and control participants $(68 \%$ vs. $69 \%$ respectively refilled at least $80 \%$ of their medication, $\mathrm{p}=\mathrm{NS}$ ). The web group demonstrated significantly higher adherence to four weeks of acute treatment compared to controls $(73 \%$ vs. $42 \%, p=0.003)$. There were significantly greater improvements in web group participants in IBD knowledge, disease-specific quality of life $(p=0.04)$, general health $(p=0.009)$, vitality $(p=0.03)$, and emotional $(p<0.0001)$ and social functioning $(\mathrm{p}=0.002)$ as compared with the control group. Half of all participants experienced at least one flare of symptoms during the study period, with no difference in flare rates between the web and control groups. However, relapses were significantly shorter in the web group compared to the control group (18 days vs. 77 days, $\mathrm{p}<0.0001$ ). There was otherwise no difference between the groups in disease activity scores as measured by the SCCAI, or in the rate of hospitalizations.

There were a few differences seen in the Irish arm of the study. Medication adherence to four weeks of treatment was significantly greater in the web group compared to controls $(73 \%$ vs. $29 \%, p=0.03)$. There were no differences between study groups in terms of IBD knowledge or disease-specific quality of life. The web group demonstrated improved mental health $(p=0.01)$, physical functioning $(p=0.03)$, and social functioning $(p=0.02)$ compared to controls. The web group experienced a higher frequency of relapses; however, these were shorter than the relapses experienced by controls ( 30 days vs. 70 days, $p<0.03$ ).

A cost analysis revealed that UC-related acute visits were higher in the control group compared to the web group (107 vs. 21 visits, $p<0.0001)$. There were also fewer routine visits in the web group. Conversely, there were a significantly higher number of emails (86) and phone calls (21) from web participants than from controls (7 emails and 17 phone calls). Fecal calprotectin was measured in both the Dutch and Irish arms of the study to serve as an objective measure of inflammation. Overall, a lower proportion of web group participants had an elevated fecal calprotectin level compared to controls.

Overall, $88 \%$ of participants in the web group preferred telemedicine to conventional care. Participants felt empowered by the ability to initiate treatment using the web-guided 
solution and safe with the option of a web-based follow-up with their provider. The authors proposed that a web-based treatment strategy such as this could reduce patients' dependency on doctors as well as mitigate health care costs. However, like the study by Cross et al., this study also had a high attrition rate over one year of follow up.

In summary, telemedicine is well accepted by patients with IBD, including patients with UC. Longitudinal use of these systems seems feasible; however the high attrition rates reported in both randomized, controlled trials raises concerns about long term adherence to telemonitoring systems. In addition to showing high rates of patient acceptance and feasibility, available studies have demonstrated improvements in clinical outcomes. The quasiexperimental study by Cross et al. reported decreased disease activity, improved quality of life, and increased knowledge after use of IBD HAT for 6 months. Similarly, both randomized controlled trials of telemedicine for UC showed improvements in disease activity as measured by disease activity indices or fecal calprotectin levels and improvements in quality of life. However, flare rates were increased in the European study. Despite the higher rate of flares in the telemedicine arm, the length of flares was less in the telemedicine group. Despite improvements in disease activity, adherence was not consistently better in the telemedicine arms in either study, except in the acute treatment phase of the study by Elkjaer et al. Quality of life improved in the telemedicine arms of both studies and utilization of health care resources was less in the telemedicine group in the European study (although email and phone calls increased in the telemedicine group). Therefore, the evidence thus far in IBD and UC, demonstrates a positive effect of telemedicine on clinical outcomes. Further studies are needed to confirm these results and to study the $\mathrm{CD}$ population. Further, larger studies are needed to explore subgroups that might particularly benefit from telemedicine, specifically patients with decreased access to care, a history of nonadherence, poor social support, more severe disease (moderate to severe IBD), patients with active disease vs. disease in remission, and patients initiating new drug therapy. Lastly, the financial impact of telemedicine on IBD care, positive or negative, needs to be explored.

\section{Irritable bowel syndrome}

Irritable bowel syndrome (IBS) is chronic, functional gastrointestinal condition characterized by chronic intermittent abdominal pain associated with an alteration in bowel movements. The change in bowel movements can be in frequency (diarrhea or constipation) or a change in stool formation (liquid stools, hard stools, unusual stool shapes). Patients often have associated bloating and worsening of symptoms during stress. IBS differs from IBD in that there is no inflammatory component, and endoscopy and biopsy results are normal Nonetheless, the chronic symptoms of IBS can be quite distressing to patients, decrease quality of life, and result in a high rate of work absenteeism.

A German group set out to investigate whether an open-access Internet-based questionnaire on gastrointestinal symptoms and quality of life could be used to collect data from patients with IBS (Enck et al., 2006). To accomplish this, investigators created an abbreviated symptom questionnaire modified from the IBS Rome II modular questionnaire, a validated questionnaire for IBS (Drossman et al, 2000, as cited in Enck et al., 2006). This was done to reduce estimated completion time from 30 minutes to 5 minutes. They also used a validated 22-item general quality of life questionnaire that took 10 minutes to complete. Both questionnaires were available online over a 43-month period. A total of 5,256 individuals 
completed the questionnaires, with an average of 100 respondents per month. Data from 850 individuals were excluded due to incomplete responses. Two thirds of respondents were women. The mean age of participants was 38 years. The investigators determined that among all respondents, $61 \%$ met criteria for the diagnosis of IBS. Verification of the diagnosis of presumed participants with IBS was not performed. A commentary on this study was published two years later by Muth and Switzer in the European Journal of Gastroenterology that identified concerns with its validity (Muth \& Switzer, 2008). They argued that while Enck's group used a validated questionnaire to assess quality of life, the questionnaire had been validated for pen-and-paper use only. Muck and Switzer also commented on the abbreviated survey tool used to assess gastrointestinal symptoms. While the survey had content validity since it was based on the standard Rome criteria for the diagnosis of IBS, the impact of having reduced items on this questionnaire was not assessed. In addition, it was unclear in what language the tools were administered. Nevertheless, Enck et al. did demonstrate that completion of an IBS and quality of life instrument electronically was feasible in an IBS population.

While the previous study solely aimed to examine the role of the Internet in data collection, a randomized control trial was performed in Sweden to investigate whether cognitive behavior therapy delivered via the Internet could be effective in managing patients with IBS (Ljotsson et al., 2010). Eighty-five self-referred participants were recruited to participate in the trial between May 2008 and July 2008. Self-referrals were generated from several online Swedish discussion forums for patients with IBS, a major newspaper article, and a Stockholm clinic that specialized in IBS care. Only patients with a diagnosis of IBS confirmed by Rome criteria were included in the study. Exclusion criteria included: a) initiation of symptoms after age 50, b) blood in the stool without history of hemorrhoids, c) diarrhea-predominant IBS without colonoscopic evaluation, d) rapid weight loss not explained by diet, e) nocturnal symptoms causing sleeplessness, and f) less than 2 years of IBS symptoms. Participants were also excluded if they had any previous history of IBD, celiac disease, or lactose intolerance. The GI Symptom diary (Blanchard, 2001, as cited in Ljotsson et al., 2010) was used to measure symptom severity and the Irritable Bowel Syndrome Quality of Life Instrument (Patrick et al, 1998, as cited in Ljotsson et al., 2010) for disease-specific quality of life, respectively. These questionnaires were used for pre- and post-intervention testing.

A total of 83 participants completed pre-testing then were randomized to either the treatment or control group. The 42 participants assigned to the treatment group received a 10-week cognitive behavioral therapy (CBT) protocol, which consisted of a five-step selfhelp manual presented on printer-friendly web pages. The five steps in the CBT manual guided participants through a series of mindfulness exercises in which they were encouraged to be immediately aware of their gastrointestinal symptoms, thoughts, feelings, behaviors, and impulses. The fifth step covered a series of exposure exercises, in which participants were to experience situations that would likely provoke symptoms such as eating trigger foods or attending a meeting with tight clothes that would provoke pain. They were encouraged to use mindfulness exercises during these exposure exercises. Participants in the treatment group had access to an online discussion forum to discuss the treatment with other participants and to communicate with a graduate psychology student trained in CBT over the course of the treatment period.

The 43 participants randomized to the control group were placed on a waiting list for CBT. The authors gave control participants access to a separate online discussion forum and allowed participants to contact a psychology student. However, the student did not provide 
CBT-based advice for treatment of IBS symptoms. After completing post-testing, participants in the control group were then invited to cross over into the treatment arm to receive CBT. In the treatment group, $74 \%$ of participants reached the fifth step in the CBT manual. Four participants never completed the first step. Participants in the treatment group reported significant improvement in abdominal pain, bloating, flatulence, and IBS-specific quality of life. Effects on diarrhea, constipation, nausea, or belching were small or insignificant. Small but significant improvements in depressive symptoms and gastrointestinal symptoms were observed in the control group from baseline.

There were several limitations to the study. First, since participants were self-referred, it is possible that participants were not representative of the IBS community (better educated, higher motivation, etc.). Second, the precise role of telemedicine on the improved outcomes in this study is not known as the control group did not receive CBT in any form. A comparison arm of patient treated with conventional CBT would have been useful. Nevertheless, this study provided evidence that the Internet is a viable method of delivery of CBT for a select IBS population. Further studies on this delivery method, as well as on the content of treatment delivered, are warranted.

In summary, there are limited studies available on the use of telemedicine for patients with IBS. The data available demonstrates that patients with IBS can complete web-based questionnaires and that therapy using a web-based system is feasible and effective in a select IBS patient population. Whether other forms of IBS therapy (medical therapy or other forms of psychotherapy) can be delivered electronically to improve outcomes is not known at this time. Further, it is not clear that telemedicine for IBS improves outcomes compared to standard of care or best available care. Lastly, the ability of telemedicine to decrease health care costs in IBS has not been assessed.

\section{Chronic hepatitis $C$ infection}

Chronic hepatitis $\mathrm{C}$ virus (HCV) infection is another chronic disease process in the field of gastroenterology in which telemedicine has been studied. As the incidence and prevalence of HCV infection rises in the United States, access to care becomes a barrier in providing adequate treatment. According to the Centers for Disease Control, HCV infection is the most common chronic blood-borne infection in the United States; approximately 3.2 million persons are chronically infected. Anti-viral treatment reduces the risk of disease progression to cirrhosis and liver cancer, both potentially fatal outcomes of this infection. Liver transplantation remains a limited and costly option, and is only applicable in the final stages of disease. Telemedicine consulting with videoconferencing has been described as a method to connect practitioners in the rural setting and in correctional facilities with gastroenterologists and hepatologists at tertiary care facilities in order to improve access to care and anti-viral therapy.

The first description of this type of telemedicine application emerged in 1994. The University of Alberta was identified as the center for a nation-wide, free-physician consultation service in which practitioners in rural areas across Canada were given immediate access to a gastroenterologist by phone, fax, or email (Rafuse, 1994). The project was designed to offer consultation on the treatment and management of hepatitis. Although expectations were high about the positive impact of this project on hepatitis patient outcomes in Canada, no qualitative or quantitative data were published revealing the effect of this particular intervention. 
Nine years later, an abstract presented at the 2003 Digestive Diseases Week conference entitled "Application of Telemedicine in the Management of Chronic Hepatitis C in Texas Prisons" summarized the experience of applying telemedicine in the treatment of $\mathrm{HCV}$ in the correctional setting (Lau, Fehmi, \& Sifuentes, 2003). Upon screening, correctional facility physicians discussed patient laboratory results and medical conditions with hepatologists via telemedicine prior to initiation of anti-viral treatment. Regular telemedicine clinics were scheduled during the treatment period to review laboratory results and medication side effects. 470 patients completed six months of therapy, with $52 \%$ of patients being Caucasian, 18\% African American, and 30\% Hispanic. Among those with genotype 1 virus, 33\% demonstrated treatment response as compared with $71 \%$ with non-genotype $1 \mathrm{HCV}$. The presenters concluded that telemedicine was an effective means of managing the treatment of chronic $\mathrm{HCV}$ in the correctional health system given that these response rates were comparable with those in the general community.

That same year the University of California, Davis (UCD) published a cohort study describing patient and provider acceptance of their telemedicine program created to aid in the management of patients with HCV (Rossaro et al, 2003). The UCD telemedicine program encompassed a large referral network of over 60 sites throughout the state of California. Telecommunication methods used through this program included T1, frame relay and the Integrated Services Digital Network. Relevant clinical information such as laboratory testing, imaging, and liver biopsy reports were required to be sent for review by the specialist prior to each hepatology consultation. Primary care providers were invited to participate during the consultation, often performing the physical exam as instructed by the specialist, which added a distance learning component to the consultation. In some situations, a "patient exam" camera was used to magnify details, such as skin changes consistent with liver disease like spider angiomata or palmar erythema.

After each telemedicine consultation, patients and providers completed a satisfaction survey. Both parties indicated if they would choose to continue using the telemedicine service or return to standard care. The authors did not disclose how many surveys were sent, but 22 patient surveys and 20 provider surveys were returned from 5 clinics. Satisfaction was scored on a 5-point scale that ranged from 1 representing "Poor" to 5 representing "Excellent." Overall satisfaction scores were high among patients and providers, with mean scores ranging from 4.7 to 4.95 for items such as overall satisfaction with the system, ability to understand consultant recommendations, and quality of the audio and video signal from the telemedicine equipment. All patients indicated they would prefer to continue using telemedicine in the future, and all providers also reported they would recommend use of telemedicine services in the future. This is another example where telemedicine offered the opportunity to improve access to specialized care for the treatment of HCV to patients in rural areas and correctional facilities, while providing educational opportunities for primary care providers.

In 2007, the UCD group published a follow-up quasi-experimental study, comparing the impact of multi-point videoconferencing versus standard lecturing to primary care providers (Rossaro et al 2007). Participants included primary care physicians, nurse practitioners, physician assistants, and registered nurses. Prior to the educational intervention, participants completed a single page questionnaire, documenting certain demographic information such as their total number of $\mathrm{HCV}$ patients seen, year of graduation from professional training, and attendance at Continuing Medical Education 
(CME) conferences within the previous 6 months. The outcome of interest in this study was improved knowledge of the history, diagnosis, and treatment of hepatitis $C$, measured by a quiz administered before and after the educational intervention. This quiz was based on a review of current literature and guidelines for the treatment of hepatitis $\mathrm{C}$. The investigators hypothesized that telemedicine conferencing would yield knowledge outcome measures comparable with traditional teaching methods.

A board certified gastroenterologist delivered the educational intervention with identical outlines and slide materials for both the standard teaching and the videoconferencing groups. To ensure consistency of teaching, the same individual delivered the intervention for both study groups. One hundred and seventy-five providers from 14 different sites met criteria to be included in the study. There were no statistically significant differences between study groups in terms of background, training, experience, or recent CME attendance. Most providers were in practice over 10 years since graduation from professional training, and more than half had seen 10 or fewer patients with hepatitis $C$.

In both study groups, pre-testing knowledge scores did not differ significantly between the three disciplines of training, but there was a significantly higher level of pre-test knowledge in the standard teaching group as compared with the videoconferencing group $(p<0.05)$. While an improvement in HCV knowledge was seen in all learners in both groups, a statistically greater improvement in knowledge scores was measured in post-testing among physicians and registered nurses in the videoconferencing group as compared with the standard teaching group $(p<0.001)$. These results suggest that videoconferencing is at least as equivalent if not better than standard CME teaching.

The following year, Rossaro's group reported results from a qualitative retrospective analysis, describing characteristics from the population of patients who received care at the Peach Tree Clinic, located in rural northern California (Rossaro et al., 2008). Over the span of seven years from 2000-2007, one hundred and three patients with HCV were treated via telemedicine. Among those treated, 37\% had cirrhosis and 2 were listed for liver transplant, although neither survived long enough to undergo transplantation. Sixty-four percent of patients at this clinic were treatment-naïve; treatment was recommended for $23 \%$ of these patients. Rossaro's group concluded that this descriptive analysis confirmed that there are a high proportion of patients with advanced liver disease due to HCV in rural California, many of whom may be candidates for treatment. They also suggested that consultation with specialists via telemedicine may help providers identify candidates for liver transplantation. Around the same time period, a study from the University of New Mexico, School of Medicine in Albuquerque, was published describing the Project Extension for Community Healthcare Outcomes (ECHO) (Arora et al., 2007). This telemedicine and distance-learning project aimed to link health care providers in rural clinics, the Indian Health Service, and prisons with specialists at the University of New Mexico. At the time, 32 of 33 counties in New Mexico were listed as medically underserved, and 14 counties were designated as areas with health professional shortage. Only $20 \%$ of New Mexico physicians practiced in rural or frontier areas. Project ECHO used teleconferencing, videoconferencing, Internet-based assessment tools, online presentations, telephone, fax, and email communications to connect primary care providers with specialists, creating centers of excellence for $\mathrm{HCV}$ care in areas that previously had very limited options for such care. Inclusion criteria for participants in this project were Internet access, use of telephone service (including fax and speaker phone functions), and the ability to view word processing documents and presentations. Partner 
organizations were recruited to participate in the Project ECHO network at state-wide health care conferences and through presentations and partner contacts. Once a provider joined the network, a member of the Project ECHO team conducted an on-site, one day training session to familiarize partners with the project. Network clinicians included primary care physicians, nurse practitioners, physician assistants, and pharmacists. These clinicians were invited to spend two days at the University of New Mexico Hepatitis Clinic and to complete an orientation and training on the Project ECHO system. They were subsequently able to present their HCV cases and related questions in a weekly 2-hour telemedicine clinic in collaboration with specialists in gastroenterology, infectious disease, psychiatry, substance abuse, and pharmacology. Clinicians who participated in Project $\mathrm{ECHO}$ also had the opportunity to become eligible for certification in HCV treatment after managing 20 patients with HCV through one-full year of antiviral therapy.

Between 2003 and 2006, 173 clinics were initiated with 1,843 cases presented and 2,997 hours of continuing education credits issued to staff. In addition, 226 patients were treated with anti-viral therapy. The investigators proposed that the Project ECHO model could be applied to the management of many different chronic conditions and that it showed great promise to improve care to people living in medically underserved areas.

More recent data on telemedicine in HCV management was presented as an abstract at the American Association for the Study of Liver Diseases conference in 2009 entitled "Use of telemedicine and the 'warm line' for the treatment of HCV infection in the correctional setting to reduce barriers to specialty care" (Nachin et al., 2009). In this abstract, investigators set out to identify, triage, and treat patients with $\mathrm{HCV}$ and estimate the prevalence of cirrhosis and end stage liver disease among inmates at the California Department of Corrections and Rehabilitation facility. To this end, the University of California, San Francisco Correctional Medicine Consultative Network held a HCV training conference in October of 2008. Primary care physicians referred patients for consultation with hepatologists via telemedicine, and a "warm line" was established to further assist primary clinicians managing HCV. This warm line was available weekdays during business hours, and responses were made within 24 hours of the request. Overall, 441 new patient consultations were performed via telemedicine between May 2008 and May 2009. The presenters concluded that use of telemedicine and the warm line offered efficient access to specialty care in this population.

In June 2011 The Project ECHO group published additional data in the New England Journal of Medicine which confirmed the utility of their model as a means to increase access to HCV treatment (Arora et al, 2011). In this prospective cohort study, outcomes were compared between patients who received HCV treatment at either the University of New Mexico (UNM) HCV specialty clinic (146 patients) or at one of 21 ECHO telemedicine sites located in primary care clinics in rural areas or prisons in New Mexico. There were a few baseline differences between the UNM clinic group and the ECHO telemedicine site groups. Specifically, patients treated at the ECHO sites were more likely to be male ( $96 \%$ of patients treated in the prison system were men). ECHO patients were also more likely to be Hispanic, and had higher mean values for weight and BMI. Despite this, there was no significant difference in sustained viral response between the two patient groups. This confirmed that HCV treatment can be delivered effectively via telemedicine in non-specialty care facilities that would otherwise have no means for delivering such care.

In summary, telemedicine has been shown to be a useful medium for communication between specialists, primary providers, and patients with HCV infection in rural areas and correctional facilities. The use and study of telemedicine in the HCV population is focused 
on providing guidance and training of primary care providers by hepatologists, in order to encourage them to provide anti-viral therapies to patients in those settings where hepatologists are unavailable. Whether telemedicine improves outcomes compared to standard of care in patients with $\mathrm{HCV}$ undergoing treatment and whether telemedicine reduces health care costs is speculative at this time.

\section{Conclusion}

Chronic gastrointestinal diseases are associated with significant morbidity and cost in the United States and throughout the world. Because of chronic nature of these diseases, the need for ongoing monitoring of flares of disease and the need for medications to control symptoms and prevent relapses, outcomes can be suboptimal. Telemedicine systems offer a modern, alternative monitoring/treatment method that can improve clinical outcomes, reduce time to initiation of treatment, increase patient access to treatment, improve patient and provider education, and reduce health care costs. The common thread present in all the studies summarized in this review is that when considering the management of chronic gastrointestinal disease, telemedicine is feasible and well accepted among various patient populations, improves access to care, and increases patients' sense of empowerment. Technology will continue to advance in quality and ease of use of telemedicine systems and will likely incorporate the use of hand-held devices. This progress should result in the increased use of telemedicine as a treatment alternative or as an adjunctive component to disease management in chronic digestive diseases.

\begin{tabular}{|c|c|c|c|}
\hline Author and Year & Disease State & $\begin{array}{l}\text { Telemanagement } \\
\text { Communication }\end{array}$ & Outcomes \\
\hline Castro, 2006 & $\begin{array}{l}\text { Inflammatory bowel } \\
\text { disease }\end{array}$ & Physician-to-patient & $\begin{array}{l}\text { Feasible method, improved } \\
\text { patient empowerment }\end{array}$ \\
\hline Cross, 2006 & $\begin{array}{l}\text { Inflammatory bowel } \\
\text { disease }\end{array}$ & Physician-to-patient & $\begin{array}{l}\text { Feasible method, excellent } \\
\text { patient acceptance of } \\
\text { technology }\end{array}$ \\
\hline Cross, 2007 & $\begin{array}{l}\text { Inflammatory bowel } \\
\text { disease }\end{array}$ & Physician-to-patient & $\begin{array}{l}\text { Feasible method, excellent } \\
\text { patient acceptance, improved } \\
\text { disease activity, quality of life } \\
\text { and patient knowledge }\end{array}$ \\
\hline Cross, 2009 & Ulcerative colitis & Physician-to-patient & $\begin{array}{l}\text { Feasible method, excellent } \\
\text { patient acceptance of } \\
\text { technology }\end{array}$ \\
\hline Cross, 2011 & Ulcerative colitis & Physician-to-patient & $\begin{array}{l}\text { High attrition rate in } \\
\text { telemanagement arm, } \\
\text { improved patient quality of } \\
\text { life compared to best available } \\
\text { care control group }\end{array}$ \\
\hline Elkjaer, 2010 & Ulcerative colitis & Physician-to-patient & $\begin{array}{l}\text { Feasible method, excellent } \\
\text { patient acceptance of } \\
\text { technology }\end{array}$ \\
\hline Elkjaer, 2010 & Ulcerative colitis & Physician-to-patient & $\begin{array}{l}\text { Improved medication } \\
\text { adherence, quality of life, and } \\
\text { patient knowledge; shorter } \\
\text { flares; less clinic visits; more } \\
\text { electronic communication in } \\
\text { web group }\end{array}$ \\
\hline
\end{tabular}




\begin{tabular}{|l|l|l|l|}
\hline Author and Year & Disease State & $\begin{array}{l}\text { Telemanagement } \\
\text { Communication }\end{array}$ & Outcomes \\
\hline Enck, 2006 & Irritable bowel syndrome & Physician-to-patient & $\begin{array}{l}\text { Internet-based questionnaire } \\
\text { administration is feasible }\end{array}$ \\
\hline Krier, 2010 & $\begin{array}{l}\text { Inflammatory bowel } \\
\text { disease }\end{array}$ & Physician-to-patient & $\begin{array}{l}\text { Patient satisfaction comparable } \\
\text { to face-to-face interaction, } \\
\text { similar interaction times } \\
\text { between physician and patient }\end{array}$ \\
\hline Ljótsson, 2010 & Irritable bowel syndrome & Physician-to-patient & $\begin{array}{l}\text { Significant improvement in } \\
\text { symptoms and quality of life } \\
\text { in treatment group compared } \\
\text { to control group }\end{array}$ \\
\hline Rossaro, 2003 & Hepatitis C & $\begin{array}{l}\text { Physician-to-physician and } \\
\text { patient }\end{array}$ & $\begin{array}{l}\text { High acceptance of method } \\
\text { among patients and local } \\
\text { providers }\end{array}$ \\
\hline Rossaro, 2007 & Hepatitis C & Physician-to-physician & $\begin{array}{l}\text { Improvement in hepatitis C } \\
\text { knowledge among local } \\
\text { providers }\end{array}$ \\
\hline Arora, 2011 & Hepatitis C & Physician-to-physician & $\begin{array}{l}\text { Equal sustained virologic } \\
\text { response rate in telemedicine } \\
\text { group compared to tertiary } \\
\text { referral center }\end{array}$ \\
\hline
\end{tabular}

Table 1. Summary table of key gastrointestinal telemanagement studies and outcomes

\section{Acknowledgement}

T32 DK067872 Research Training in Gastroenterology.

\section{References}

Arora S, Thornton K, Murata G, Deming P, Kalishman S, Dion D, Parish B, Burke T, Pak W, Dunkelberg J, Kistin M, Brown J, Jenkusky S, Komaromy M, \& Quals C (2011). Outcomes of Treatment for Hepatitis C Virus Infection by Primary Care Providers. New England Journal of Medicine. June 2011; [doi:10.1056/NEJMoa1009370]Castro HK, Cross RK, \& Finkelstein J (2006). Using a Home Automated Telemanagement (HAT) system: experiences and perceptions of patients with inflammatory bowel disease. Proceedings of American Medical Informatics Association Annual Symposium. 2006:872.

Crohn's and Colitis Foundation of America. http:/ / www.ccfa.org. 2010

Cross RK, Arora M, \& Finkelstein J (2006). Acceptance of telemanagement is high in patients with inflammatory bowel disease. Journal of Clinical Gastroenterology. March 2006; 40(3):200-8.

Cross RK, Finkelstein J (2007). Feasibility and acceptance of a home telemanagement system in patients with inflammatory bowel disease: a 6-month pilot study. Digestive Diseases and Sciences. February 2007; 52(2):357-64.

Cross RK, Cheevers N, \& Finkelstein J. (2009) Home telemanagement for patients with ulcerative colitis (UC HAT). Digestive Diseases and Sciences. November 2009; 54(11):2463-72.

Cross RK, Cheevers, N, Rustgi, A, Langenberg, P, \& Finkelstein J (2011). A Randomized, Controlled Trial of Home Telemanagement in Patients with Ulcerative Colitis (UC 
HAT). Proceedings of American Gastroenterological Association Digestive Diseases Week, Chicago. May 2011.

Elkjaer, M, Burisch, J, Avnstrom, S, Lynge, E, \& Munkholm, P. Development of a Web-based concept for patients with ulcerative colitis and 5-aminosalicylic acid treatment. European Journal of Gastroenterology and Hepatology. 2010; 22(6):695-704

Elkjaer M, Shuhaibar M, Burisch J, Bailey Y, Scherfig H, Laugesen B, Avnstrøm S, Langholz E, O'Morain C, Lynge E, \& Munkholm P (2010). E-health empowers patients with ulcerative colitis: a randomised controlled trial of the web-guided 'Constant-care' approach. Gut. December 2010; 59(12):1652-61

Enck P, Kowalski A, Martens U, \& Klosterhalfen S. Internet-based assessment of bowel symptoms and quality of life (2001). European Journal of Gastroenterology and Hepatology. December 2006; 18(12):1263-9.

Kane, S, Huo, D, Aikens, J, \& Hanauer, S. Medication nonadherence and the outcomes of patients with quiescent ulcerative colitis. American Journal of Medicine. 2003; 114(1):39-43

Krier M, Kaltenbach T, McQuaid K, \& Soetikno R. (2010) Broadening the Access to Specialized IBD Care Using a Consumer Grade Affordable Telemedicine System. Proceedings of American Gastroenterological Association Digestive Diseases Week. New Orleans, May 2010.

Lau T, Fehmi S, \& Sifuentes D (2003). Application of telemedicine in the management of chronic hepatitis C in Texas prisons. Proceedings of American Gastroenterological Association Digestive Diseases Week, Orlando, April 2003.

Ljótsson B, Falk L, Vesterlund AW, Hedman E, Lindfors P, Rück C, Hursti T, Andréewitch S, Jansson L, Lindefors N, \&Andersson G (2010). Internet-delivered exposure and mindfulness based therapy for irritable bowel syndrome--a randomized controlled trial. Behavioral Research and Therapy. June 2010; 48(6):531-9.

Muth ER \& Switzer FS. Internet-based assessment of bowel symptoms and quality of life. European Journal of Gastroenterology and Hepatology. June 2008; 20(6):503-5.

Nachin P, Kerbleski M, Gaglioti A, Mahoney M, Protell R, Kohler L, \& Imperial J. (2009) Use of telemedicine and the 'warm line' for the treatment of hepatitis $\mathrm{C}$ infection in the correctional setting to reduce barriers to specialty care. Proceedings of American Association for the Study of Liver Diseases, San Francisco, November 2009.

Rafuse J. (1994). University of Alberta program links northern hepatitis patients with southern consultants. Canadian Medical Association Journal. September 1994; 151(5):654-5.

Rossaro L, Tran T, Cole S, \& Nesbitt T. (2003). Telemedicine: Improving Access to Care of Hepatitis C - Guest Editorial. Practical Gastroenterology. April 2003; (27):17-22.

Rossaro L, Tran T, \& Ransibrahmanakhul K. (2007). Hepatitis C Videoconferencing: The Impact on Continuing Medical Education for Rural Healthcare Providers. Telemedicine and e-Health. 2007, 13 (3): 269-277.

Rossaro L, Aoki C, \& Yuk J. (2008) The evaluation of patients with hepatitis C living in rural California via telemedicine. Telemedicine and e-Health. December 2008;14(10):1127-9.

Sewitch MJ, Abrahamowicz, M, Barkun, A, Bitton, A, Wild, GE, Cohen, A, \& Dobkin, PL. Patient nonadherence to medication in inflammatory bowel disease. American Journal of Gastroenterology. 2003; 98(7):1535-44. 


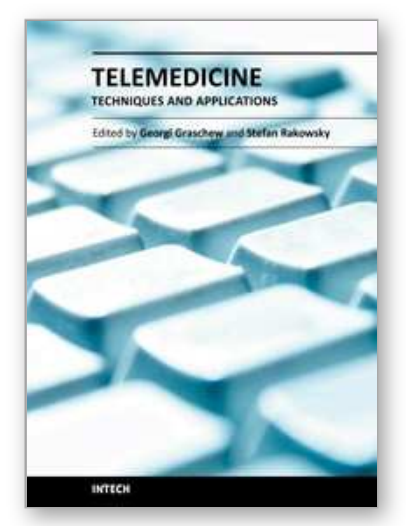

\author{
Telemedicine Techniques and Applications \\ Edited by Prof. Georgi Graschew
}

ISBN 978-953-307-354-5

Hard cover, 514 pages

Publisher InTech

Published online 20, June, 2011

Published in print edition June, 2011

Telemedicine is a rapidly evolving field as new technologies are implemented for example for the development of wireless sensors, quality data transmission. Using the Internet applications such as counseling, clinical consultation support and home care monitoring and management are more and more realized, which improves access to high level medical care in underserved areas. The 23 chapters of this book present manifold examples of telemedicine treating both theoretical and practical foundations and application scenarios.

\title{
How to reference
}

In order to correctly reference this scholarly work, feel free to copy and paste the following:

Raymond Cross and Sandra Quezada (2011). Telemedicine for Chronic Digestive Diseases: A Systematic Qualitative Review, Telemedicine Techniques and Applications, Prof. Georgi Graschew (Ed.), ISBN: 978-953307-354-5, InTech, Available from: http://www.intechopen.com/books/telemedicine-techniques-andapplications/telemedicine-for-chronic-digestive-diseases-a-systematic-qualitative-review

\section{INTECH}

open science | open minds

\section{InTech Europe}

University Campus STeP Ri

Slavka Krautzeka 83/A

51000 Rijeka, Croatia

Phone: +385 (51) 770447

Fax: +385 (51) 686166

www.intechopen.com

\section{InTech China}

Unit 405, Office Block, Hotel Equatorial Shanghai

No.65, Yan An Road (West), Shanghai, 200040, China

中国上海市延安西路65号上海国际贵都大饭店办公楼 405 单元

Phone: +86-21-62489820

Fax: +86-21-62489821 
(C) 2011 The Author(s). Licensee IntechOpen. This chapter is distributed under the terms of the Creative Commons Attribution-NonCommercialShareAlike-3.0 License, which permits use, distribution and reproduction for non-commercial purposes, provided the original is properly cited and derivative works building on this content are distributed under the same license. 